\title{
Discrete wavelet transform de-noising in eukaryotic gene splicing
} Tina P George* ${ }^{*}$ and Tessamma Thomas ${ }^{2}$

Addresses: ${ }^{1}$ Department of Electronics and Instrumentation, College of Engineering, Kidangoor, Kottayam, Kerala, India and ${ }^{2}$ Department of Electronics, Cochin University of Science And Technology, Kerala, India

E-mail: Tina P George* - tinapgcusat@gmail.com; Tessamma Thomas - tess@cusat.ac.in

${ }^{*}$ Corresponding author

from The Eighth Asia Pacific Bioinformatics Conference (APBC 20I0)

Bangalore, India $18-21$ January 2010

Published: 18 January 2010

BMC Bioinformatics 20 I0, I I(SuppI I):S50 doi: I0.1 I86/I47I-2I05-II-SI-S50

This article is available from: http://www.biomedcentral.com/I47I-2I05/II/SI/S50

(c) 2010 George and Thomas; licensee BioMed Central Ltd.

This is an open access article distributed under the terms of the Creative Commons Attribution License (http://creativecommons.org/licenses/by/2.0), which permits unrestricted use, distribution, and reproduction in any medium, provided the original work is properly cited.

\begin{abstract}
Background: This paper compares the most common digital signal processing methods of exon prediction in eukaryotes, and also proposes a technique for noise suppression in exon prediction. The specimen used here which has relevance in medical research, has been taken from the public genomic database - GenBank.

Methods: Here exon prediction has been done using the digital signal processing methods viz. binary method, EIIP (electron-ion interaction psuedopotential) method and filter methods. Under filter method two filter designs, and two approaches using these two designs have been tried. The discrete wavelet transform has been used for de-noising of the exon plots.

Results: Results of exon prediction based on the methods mentioned above, which give values closest to the ones found in the NCBI database are given here. The exon plot de-noised using discrete wavelet transform is also given.

Conclusion: Alterations to the proven methods as done by the authors, improves performance of exon prediction algorithms. Also it has been proven that the discrete wavelet transform is an effective tool for de-noising which can be used with exon prediction algorithms.
\end{abstract}

\section{Background}

Genes in eukaryotic cells have two sub-regions, exons and introns [1], depicted in Figure 1. A preliminary step in the analysis of genomic data, known as DNA-splicing or exon prediction, determines the locations of the exons. The four bases of each strand of the DNA doublehelix - Adenine, Thymine, Guanine, and Cytosine are represented distinctly in a genomic sequence with the letters A, T, C, and G to [1]. Protein-coding regions in a
DNA sequence-exons (Figure 1) exhibit a period-3 property [1] because of the codon structure involved in the translation of base sequences into amino acids $[2,3]$. The period-3 property is in general regarded as a good preliminary indicator of exon locations, although there are certain exceptions [2]. Digital Signal Processing (DSP) techniques which exploit this period 3 property for exon prediction make use of DSP tools like the Discrete Fourier transform (DFT) [4] or bandpass digital 


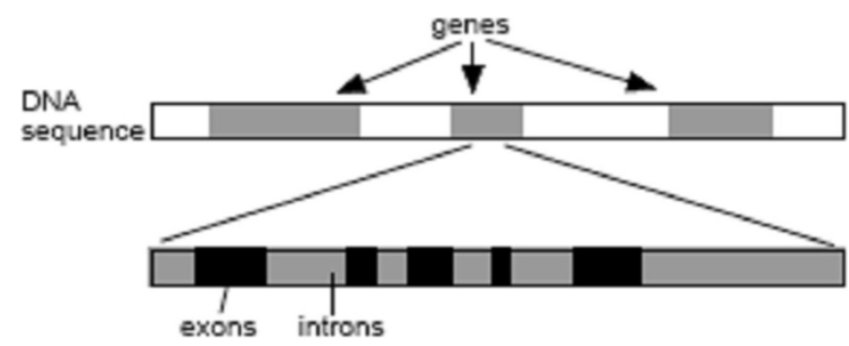

Figure I

Introns and exons in a DNA sequence. A DNA

sequence has genes as well as inter-geneic spaces(shown in white) in it. The genes in turn are made of introns and exons.

filters [5]. Trevor W. Fox and Alex Carreira [6] have proposed a method of reduced computation to map out exons in a genomic sequence, suppressing noise to a greater degree. But the drawback of all these methods is the continued presence of inter-exon noise. We have used the Discrete Wavelet Transform (DWT) to achieve greater noise suppression [7]. To design any exon prediction algorithm, first step is to convert the sequences of letters from the four-character alphabet into binary sequences conducible to digital signal processing. The numerical sequence resulting from a character string of length $\mathrm{N}$ can be written as

$$
\begin{aligned}
& x[n]=a u_{A}[n]+t u_{T}[n]+c u_{C}[n]+g u_{G}[n], \\
& n=0,1,2, \ldots \ldots \ldots . . . N-1 .
\end{aligned}
$$

$u_{A}[n], u_{T}[n], u_{C}[n]$, and $u_{G}[n]$ are the binary indicator sequences, which take the value of either 1 or 0 at location $n$, depending on whether the corresponding character exists or not, respectively, at $n$. Here we have taken values of a, $\mathrm{t}, \mathrm{c}, \mathrm{g}$ as 1 . The string ACCTG has $N=5$ and is called the length of the sequence. Also,

$$
u_{A}[\mathrm{n}]+u_{T}[\mathrm{n}]+u_{C}[\mathrm{n}]+u_{G}[\mathrm{n}]=1
$$

\section{Methods}

Many digital signal processing methods have been tried for genomic data analysis with proven results $[1,4-6,9,10]$, are but a few examples of such published work.

\section{Exon prediction using the DFT - The binary method}

This method [4] uses the binary indicator sequences obtained as described above, the DSP tool used being the DFT. As per the classical definition [11], DFT of a sequence $x[n]$, of length $N$, is itself another sequence $X$ $[k]$, of the same length $N$.

$$
X[k]=\sum_{n=0}^{N-1} x[n] e^{-j \frac{2 \pi}{N} k n}, \quad k=0,1, \ldots, N-1
$$

The sequence $X[k]$ provides a measure of the frequency content at "frequency" $k$, which corresponds to an underlying period of $N / k$ samples. Using the above definition the $U_{A}[k], U_{T}[k], U_{C}[k]$, and $U_{G}[k]$ are the DFTs of the binary indicator sequences $u_{A}[n], u_{T}[n]$, $u_{C}[n]$, and $u_{G}[n]$, respectively and then it follows that:

$$
\mathrm{X}[\mathrm{k}]=\mathrm{aU}_{\mathrm{A}}[\mathrm{k}]+\mathrm{tU}_{\mathrm{T}}[\mathrm{k}]+\mathrm{cU}_{\mathrm{C}}[\mathrm{k}]+\mathrm{gU}_{\mathrm{G}}[\mathrm{k}] ;(\mathrm{k}=0,1, \ldots \ldots \ldots \mathrm{N}-1) .
$$

As already mentioned here, $\mathrm{a}=\mathrm{t}=\mathrm{c}=\mathrm{g}=1$.

The quantity : $S[k]=\left|U_{A}[k]\right|^{2}+\left|U_{T}[k]\right|^{2}+\left|U_{C}[k]^{2}\right|+\left|U_{G}[k]\right|^{2}$

has been used as a measure of the total power spectral content of the DNA character string, at "frequency" $k$. But we've found [8] that

$$
\mathrm{S}[k]=\left|\mathrm{U}_{\mathrm{A}}[k]+\mathrm{U}_{\mathrm{T}}[k]+\mathrm{U}_{\mathrm{C}}[k]+\mathrm{U}_{\mathrm{G}}[k]\right|^{2}
$$

gives better results than the one given in equation 5 . The period-3 property of a DNA sequence implies that the DFT coefficients corresponding to $k=N / 3$ is large. Thus if we take $N$ to be a multiple of 3 and plot $S[k]$ then we should see a peak at the sample value $k=N / 3$. Instead of evaluating the DFT of a full-length sequence, DFTs of several of its subsequences, (STFT) was computed for better time domain resolution by sliding the window by one entry in the sequence.

\section{Exon prediction using the DFT - The EIIP method}

In this method, described in [11], letters of the DNA sequence $A, T, C, G$ are replaced with the electron ion interaction pseudo-potentials(EIIP) of nucleotides. If we substitute the EIIP values in $x[n]$, we get a numerical sequence the 'EIIP indicator sequence', $\mathrm{x}_{\mathrm{e}}[\mathrm{n}]$ which represents the distribution of the free electrons' energies along the DNA sequence, for A, T, C, G the values are $0.126,0.1335,0.134,0.0806$ respectively. Next, DFT is evaluated and the corresponding value of the power spectrum is

$$
\mathrm{Se}[\mathrm{K}]=|\mathrm{Xe}[\mathrm{K}]|^{2}
$$

When $\mathrm{Se}[\mathrm{k}]$ is plotted against $\mathrm{k}$, it reveals a peak at N/3 for a coding region and no such peak is observable for a noncoding region. Rectangular windows were used in this work, for evaluating the STFT by breaking up the long sequence into subsequences. 


\section{Exon prediction using digital filters}

Digital filtering methods used for identification of exons make use of the period-3 behaviour [5] coding regions. The output of an antinotch filter, with a sharp gain at the frequency $2 \pi / 3$ provides this information as a function of base location. This filter [5] has an impulse response $w$ (n) given by,

$$
w(n)= \begin{cases}e^{j \omega_{0} n} & 0 \leq n \leq N-1 \\ 0 & \text { otherwise }\end{cases}
$$

Let $H(z)$ be a narrow band bandpass or anti-notch digital filter with a sharp passband cantered at $\omega_{0}=2 \pi / 3$. With the indicator sequence $x_{G}(n)$ taken as input, let $y_{G}(n)$ denote its output. In the coding regions, the sequence $x_{G}(n)$ is expected to have a period-3 component, which means that it has large energy in the filter passband. So the output $y_{G}(n)$ should be comparatively large in the coding regions as demonstrated in Figure 2. With similar notation for the other bases, define

$$
Y[n]=\left|y_{A}(n)\right|^{2}+\left|y_{T}(n)\right|^{2}+\left|y_{C}(n)\right|^{2}+\left|y_{G}(n)\right|^{2}
$$

A plot of this function is a preliminary indicator of coding regions. Filter 2 designed by the authors [6] gives better result than this anti-notch filter-Filter 1 , called so in this paper.

Filter I

The design [5] starts by considering second order all-pass filter, $A(z)$.

$$
A(z)=\frac{R^{2}-2 R \cos \theta z^{-1}+z^{-2}}{1-2 R \cos \theta z^{-1}+R^{2} z^{-2}}
$$

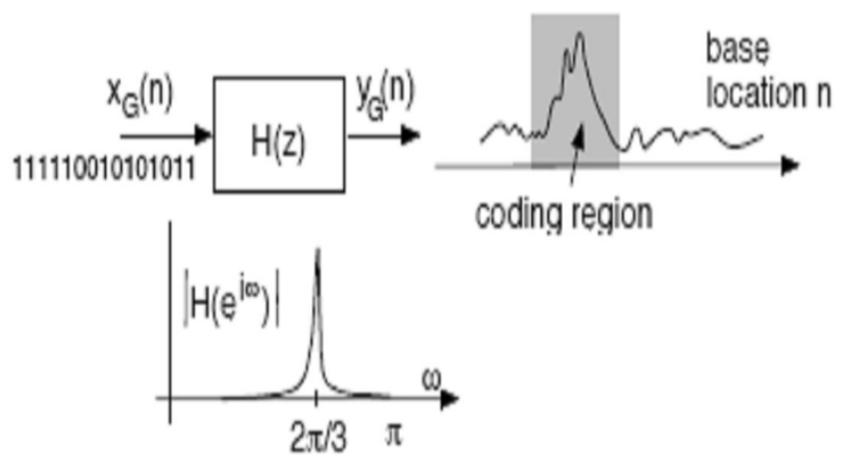

Figure 2

Expected output of anti-notch filter $x_{n}(G)$ - indicator sequence, $H(z)$-anti-notch filter with pass band centred at $2 \pi / 3, y_{n}(G)$ - output of the filter.
Now consider a filter bank with two filters $G(z)$ and $H(z)$ defined as,

$$
\left[\begin{array}{l}
G(z) \\
H(z)
\end{array}\right]=\frac{1}{2}\left[\begin{array}{cc}
1 & 1 \\
1 & -1
\end{array}\right]\left[\begin{array}{c}
1 \\
A(z)
\end{array}\right]
$$

Then $G(z)$ has the form

$$
G(z)=K\left(\frac{1-2 \cos \omega_{0} z^{-1}+z^{-2}}{1-2 R \cos \theta z^{-1}+R^{2} z^{-2}}\right)
$$

$G(z)$ is a notch filter with a zero at the frequency $w_{0}$.

$$
\begin{gathered}
{\left[\begin{array}{c}
G\left(e^{j \omega}\right) \\
H\left(e^{j \omega}\right)
\end{array}\right]=\frac{\mathrm{U}}{\sqrt{2}}\left[\begin{array}{c}
1 \\
A\left(e^{j \omega}\right)
\end{array}\right]} \\
\mathrm{H}(\mathrm{z})=(1 / 2)\left[\mathrm{z}^{-2}\left(\mathrm{R}^{2}-1\right)-\mathrm{R}^{2}+1\right] /\left[1-2 \mathrm{R} \cos \mathrm{z}^{-1}+\mathrm{R}^{2} \mathrm{z}^{-2}\right]
\end{gathered}
$$

$\mathrm{H}(\mathrm{z})$ is the required anti-notch filter with magnitude and phase responses as in Figure 3.

Filter 2

Filter 2, in this paper is an IIR single peaking filter with the peak frequency at $2 \pi / 3$. This was designed using the built-in utility of MATLAB [6]. Its magnitude and phase response is shown in Figure 4.

\section{Reduced computation technique in filter method}

The number of digital filter operations can be reduced from four to one [6] by creating a new signal that encapsulates the entire DNA sequence $u_{\mathrm{A}+\mathrm{C}+\mathrm{T}+\mathrm{G}}(n)=$ $a u_{\mathrm{A}}(n)+c u_{\mathrm{C}}(n)+t u_{\mathrm{T}}(n)+g u_{\mathrm{G}}(n)$ where $a, c, t$, and $g$ are real-valued parameters. A long DNA sequence can be

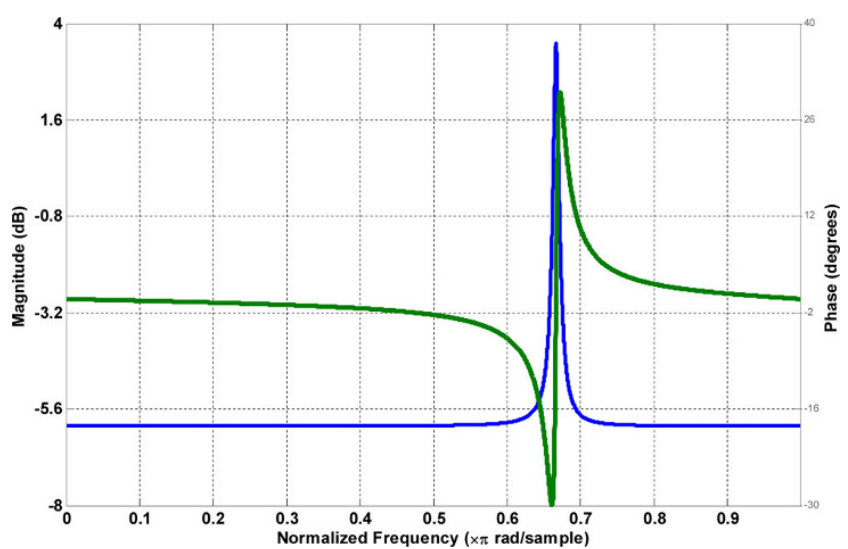

Figure 3

Magnitude and phase responses of Filter I. The magnitude response is shown in blue and phase response in green. 


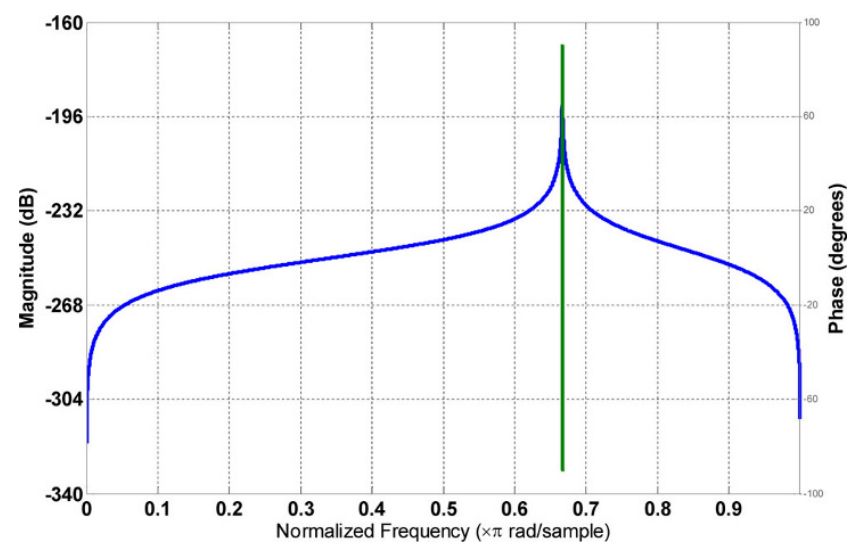

Figure 4

Magnitude and phase responses of Filter 2. The magnitude response is shown in blue and phase response in green.

approximated using a two-symbol representation, where one symbol is either $\mathrm{A}$ or $\mathrm{T}$ and the other symbol is either $\mathrm{C}$ or $\mathrm{G}$ as they are complimentary to each other. Also capitalizes on the strong periodicity exhibited by the $\mathrm{G}$ sequence. In this case, the signal becomes

$$
u_{\mathrm{T}+\mathrm{G}}(n)=t u_{\mathrm{T}}(n)+g u_{\mathrm{G}}(n) .
$$

\section{DWT to improve gene splicing techniques}

The above methods of gene splicing, though give results, better reduction in noise and accuracy of prediction is desired. The statistically optimal null filter to improve prediction of exons has been suggested by Kakumani et al. [10]. Here we've tried to improve the accuracy of a gene splicing algorithm using the Discrete Wavelet Transform (DWT). In DWT [12], the signal is passed through a series of high and low pass filters to analyze the respective frequencies followed by a scaling. The scale is changed by upsampling and downsampling (subsampling) operations. Subsampling reduces the sampling rate, or removes some of the samples of the signal. Upsampling increases the sampling rate of a signal by adds new samples. Filtering involved is explained as follows. If a signal has a maximum of $1000 \mathrm{~Hz}$ component, then half band low-pass filtering removes all the frequencies above $500 \mathrm{~Hz}$. However it is to be recalled that with discrete signals, frequency $\omega$ is expressed in terms of radians. Accordingly, the sampling frequency of the signal is equal to $2 F_{m}, \mathrm{~Hz}$, in the analog domain and $2 \pi$ radians in terms of discrete radial frequency. Therefore, the highest frequency component in a discrete signal will be $\pi$ radians. $\mathrm{Hz}$ is not appropriate for discrete signals, but used for clarity of the idea.
Decomposition of the signal into different frequency bands is obtained by successive high pass and low pass filtering of the time domain signal. The original signal $\mathrm{x}$ [n] is first passed through a halfband, highpass filter $\mathrm{g}[\mathrm{n}]$ and a lowpass filter $\mathrm{h}[\mathrm{n}]$. After the filtering, half of the samples can be eliminated ie. subsampled by 2, by discarding every other sample. This constitutes one level of decomposition, mathematically expressed as:

$$
\begin{aligned}
& y_{\text {high }}[k]=\sum_{n} x[n] \cdot g[2 k-n] \\
& y_{\text {low }}[k]=\sum_{n} x[n] \cdot h[2 k-n]
\end{aligned}
$$

$\mathrm{h}[\mathrm{n}]$ and $\mathrm{g}[\mathrm{n}]$ are the sample sequences or impulse responses and $\mathrm{y}_{\mathrm{high}}[\mathrm{k}]$ and $\mathrm{y}_{\text {low }}[\mathrm{k}]$ are the outputs of the highpass and lowpass filters, respectively, after subsampling by 2. This decomposition halves the time resolution since only half the number of samples now characterizes the entire signal. However, this operation doubles the frequency resolution, since the frequency band of the signal now spans only half the previous frequency band. The above procedure, known as subband coding, can be repeated for further decomposition. At every level, the filtering and subsampling will result in half the number of samples (and hence half the time resolution) and half the frequency band spanned (and hence double the frequency resolution). Figure 5 illustrates this procedure.

The bandwidth of the signal at every level is marked on the figure as " $\mathrm{f}$ ". The DWT of the original signal is obtained by concatenating all coefficients starting from the last level of decomposition (remaining two samples, in this case) and will have the same number of coefficients as the original signal. The difference of this from the Fourier transform is that the time localization of these frequencies will not be lost, a key advantage. Good time resolution is obtained at high frequencies, and good frequency resolution at low frequencies. All algorithms mentioned in this work were implemented using MATLAB.

\section{Results}

Figures $6,7,8,9,10,11,12,13,14$ are the results of the algorithms described in this work applied on exons in nucleotide sequence of the gene F56F11.5 of C elegans [GenBank: AF099922]. The authors have tried the DSP methods on genomic sequences of four different specimen - C elegans, Mus musculus, Sus scrofa and Homo sapien, but only the results obtained with organism $\mathrm{C}$ elegans has been included here due to lack of space. Caenorhabditis elegans is a free living nematode (roundworm), about $1 \mathrm{~mm}$ in length, which lives in temperate 


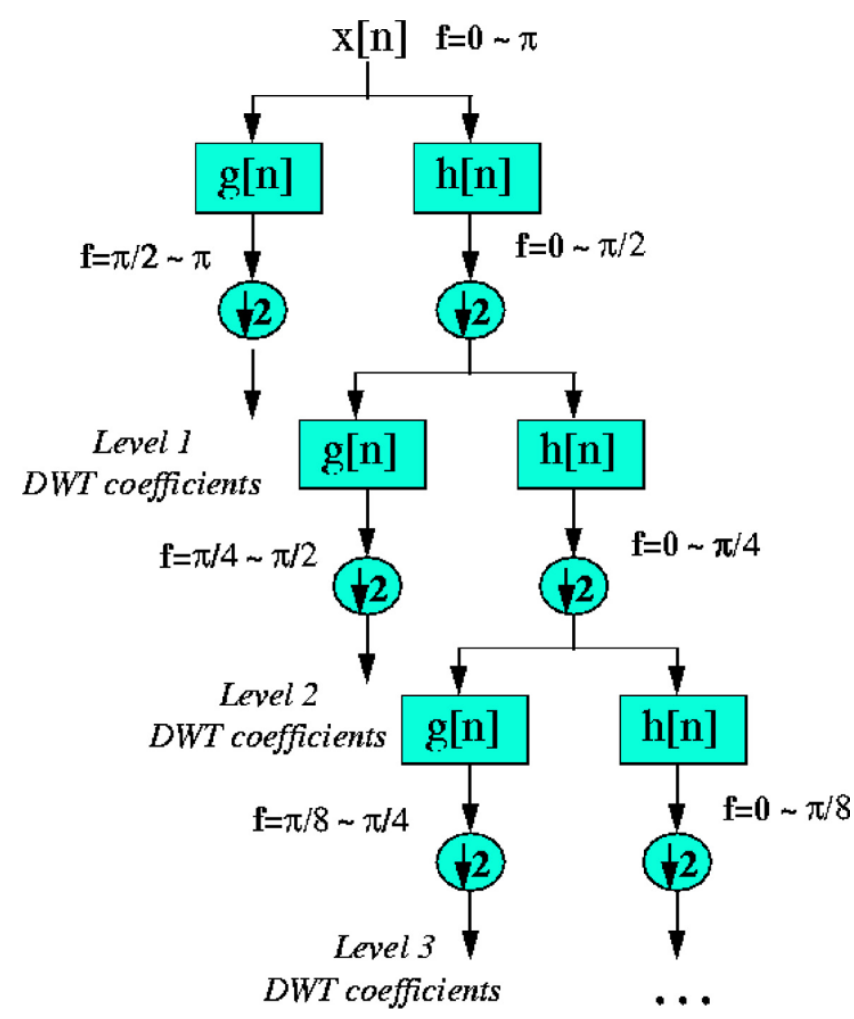

Figure 5

DWT decomposition. Schematic of DWT decomposition at 3 levels, $h[n]$ - the low pass half band filter, $g[n]$ - the high pass half band filter(notations are in discrete time domain).

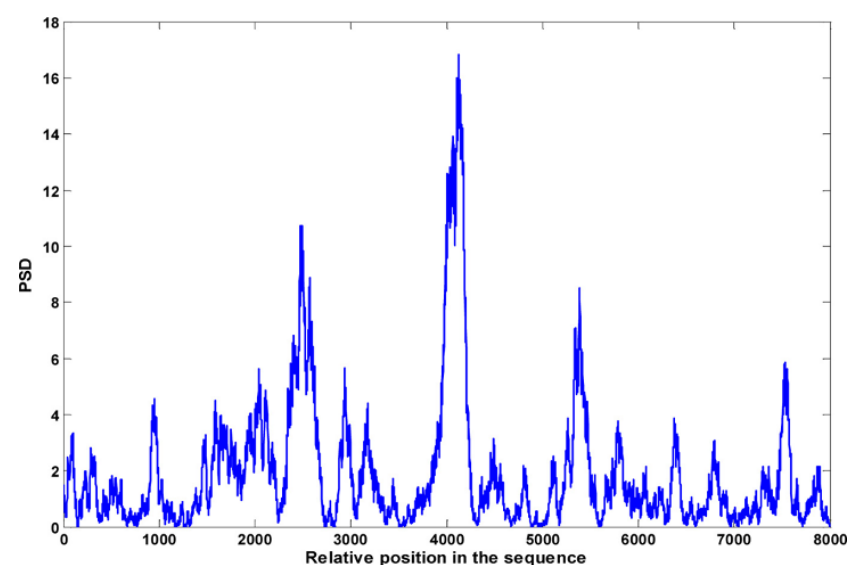

Figure 6

Exon plot-I. Result of Binary method (using the DFT), Window: 240.

soil environments. The bases are 1...42799 long and 8000 nucleotides from location 7021 have been considered which according to the NCBI data base has five exons.

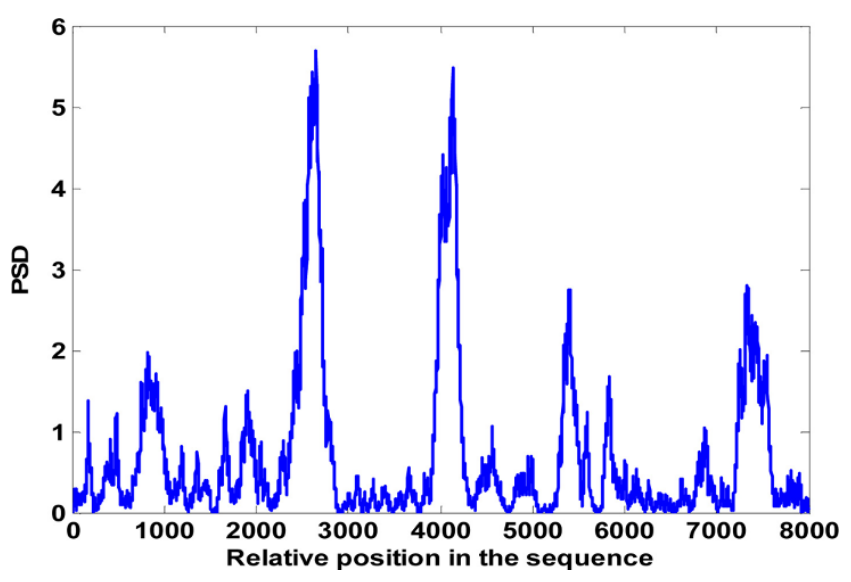

Figure 7

Exon plot 2. Result of ElIP method (using the DFT), Window: 240.

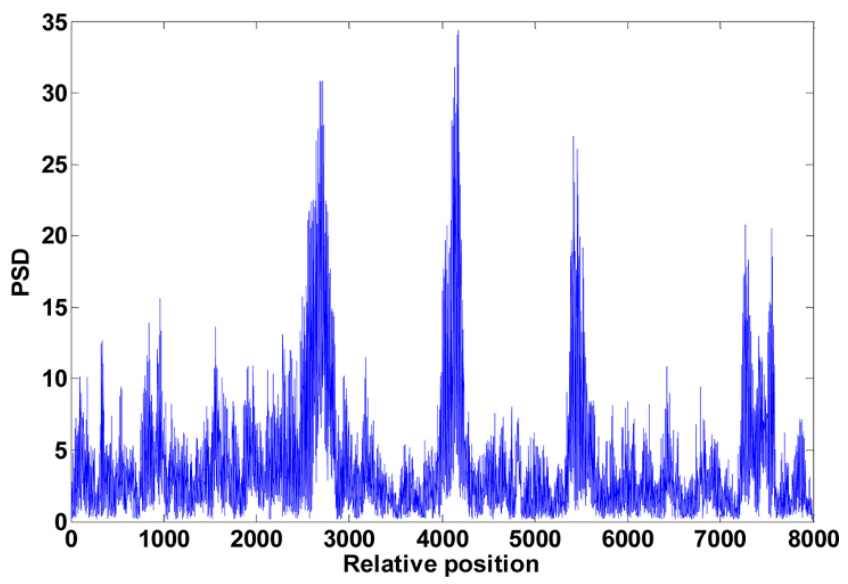

Figure 8

Exon plot 3. Result of Filter method using Filterl.

\section{Binary method and EIIP method}

Results obtained are the exon plots shown in Figures 6 and 7 respectively. Of the gene splicing algorithms mentioned here, the ones which make use of the DFT are the Binary method and the EIIP method. $\mathrm{C}$ elegans gives best result for a window length of 240 . The boundary of exons is more well defined with this window. A window size of 351 though reduces inter-exon noise, the exon boundaries tend to shift, its not shown here.

\section{Filter method}

The results obtained with digital filtering is shown in Figures 8 to 11 . The filter designed by the authors named Filter 2 here, gives better results than Filter 1[5]. The noise suppression technique with reduced computation [6] reduces inter-exon noise to a great. Exon plot 5 shows 


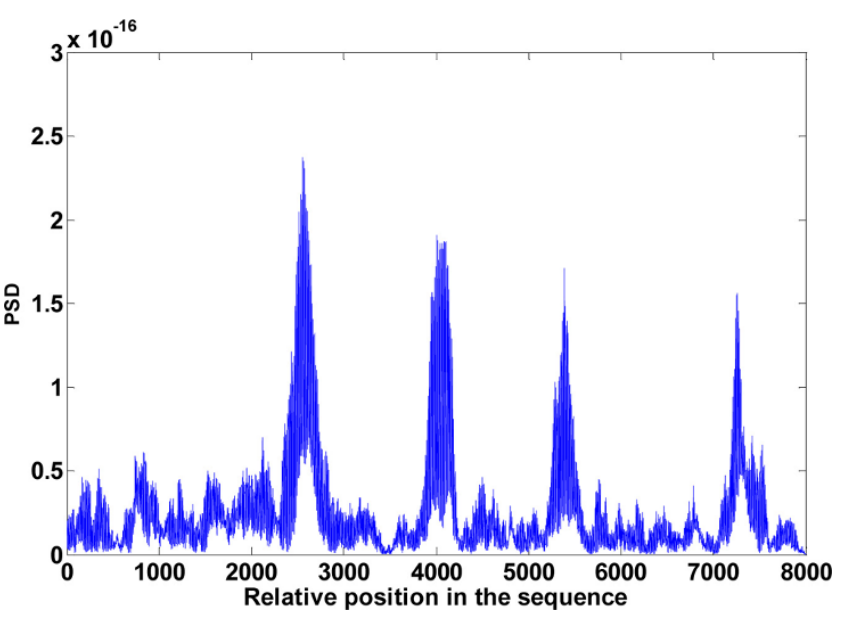

Figure 9

Exon plot 4. Result of Filter method, using Filter2, designed by the authors detailed in [8].

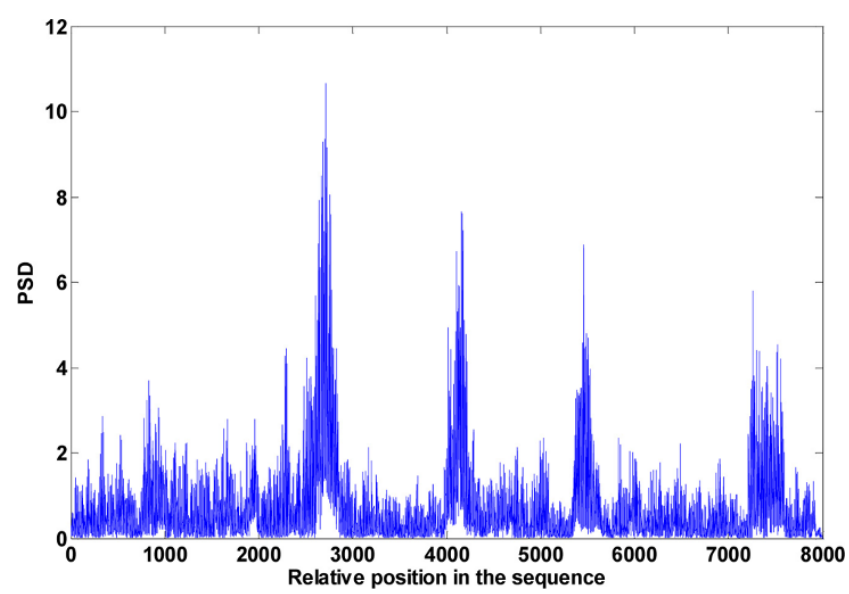

Figure 10

Exon plot 5. Result of the reduced computation method [6] using Filter I

that even the noise suppression technique when used with the filter 1 fails to give the desired results, but very effective when used in conjunction with the filter 2 as seen from exon plot 6 (Figure 11). But even then, the first exon located between 7947 and 8059, with relative position in the plot, 926 - 1079 cannot be distinguished from the surrounding inter-exon noise. The noise peaks as seen in the exon plot 5 (Figure 10, reduced computation technique with filter 2) are stronger than the half power values of the exon peaks. It's evident that these methods need improvement. Hence DWT denoising has been tried. The best results as obtained from reduced computation technique with IIR antinotch filtering using filter 2 was used for further

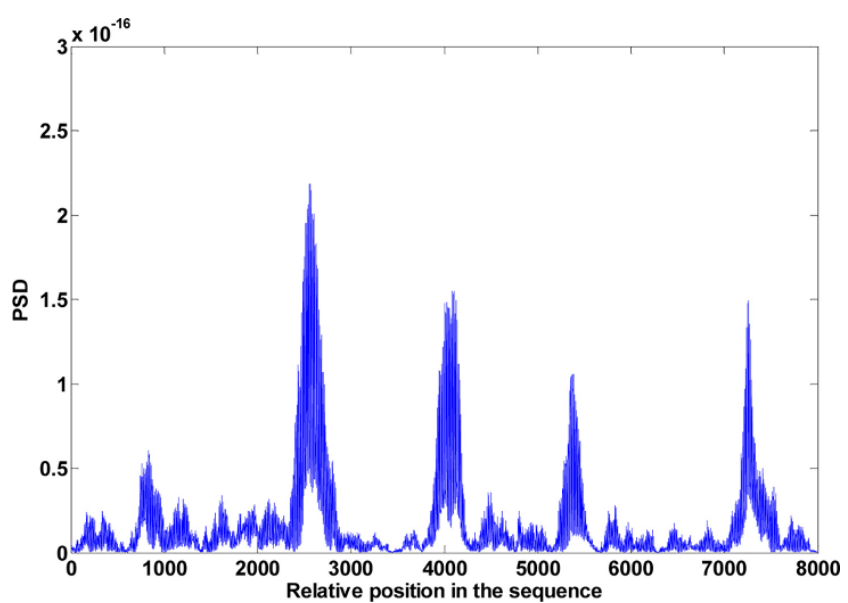

Figure II

Exon plot 6. Result of the reduced computation method [6] using Filter2

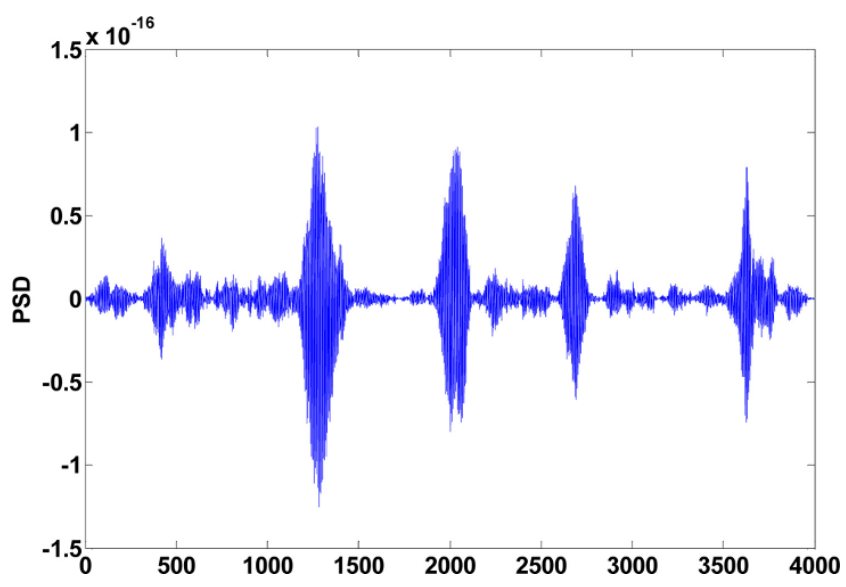

Figure 12

High frequency components of level I DWT

decomposition. The high frequency components in the spectrum after level I DWT decomposition.

treatment with DWT. All the results are shown in tabular form in table 1 against standard exon lengths give in the NCBI database.

\section{DWT to improve gene splicing techniques}

Figure 12 mentioned in the results section shows the detail coefficients and Figure 13 shows the approximation coefficients of Haar decomposition respectively. The final exon plot obtained after DWT treatment are given in Figure 14. Notice that the in exon plot 6, Figure 11 power levels corresponding to the first exon which had half power values almost equal to the noise levels (exon plot 6) has been accentuated such that there is no mistaking between exon region and intron region. As the 


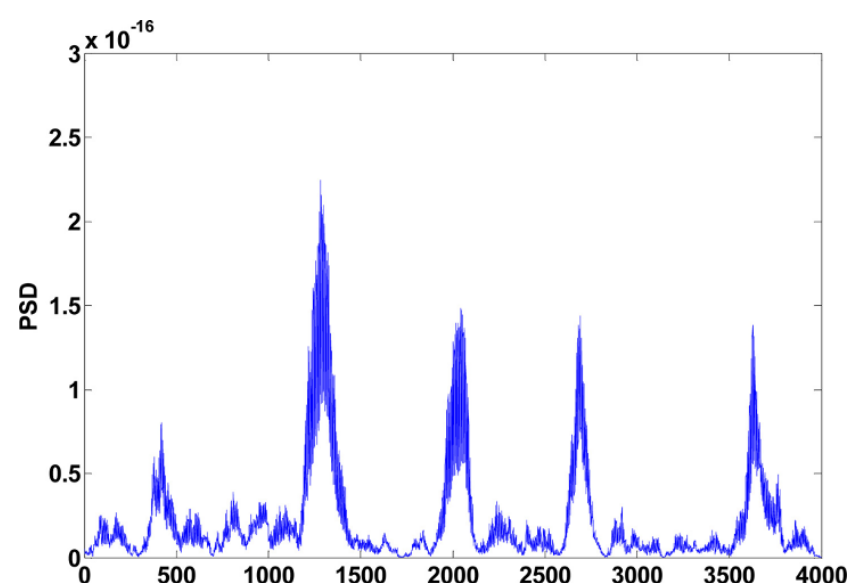

Figure 13

Low frequency components of I level DWT decomposition. The low frequency components in the spectrum after level I DWT decomposition

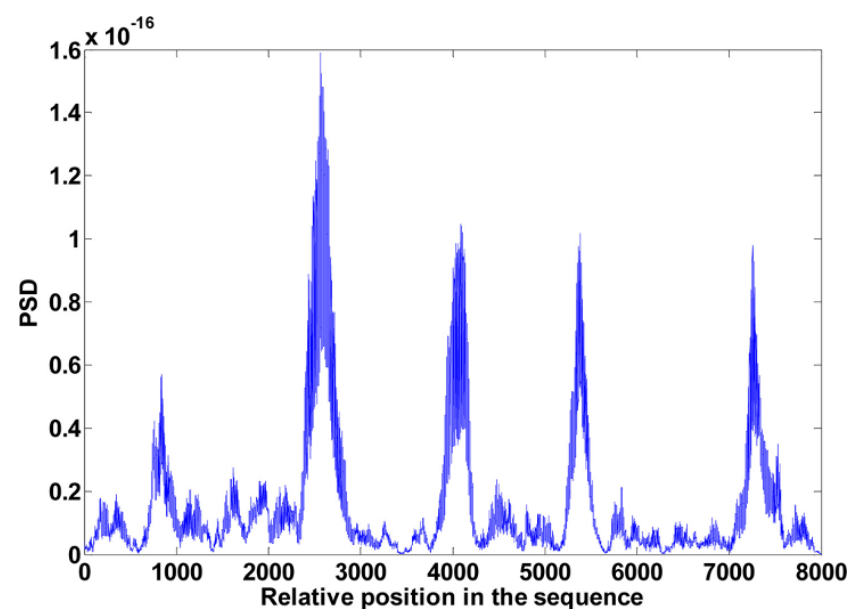

Figure 14

Exon plot after DWT de-noising. The exon plot with pronounced peaks after DWT de-noising. signals desired corresponding to exon peaks are in the lower region of the spectrum spanning the $0-\pi / 2$ range, against a discrete frequency interval of $-\pi$ to $\pi$, a single level decomposition and reconstruction was sufficient here. As already mentioned, the region of the genomic sequence of C elegans has 8000 nucleotides from 7021 to 15021 . The exon plots in figures 6 to figure 14 show 8000 nucleotide locations with the exons depicted as spectral peaks. The exon boundaries obtained after denoising with DWT are the same as those obtained with the reduced computation technique using Filter2, as the the exon plot obtained with the method was used for subsequent wavelet decomposition and re-construction. Hence the exon boundaries are not tabulated separately for the de-noised result.

\section{Discussion}

DFT is a conventional frequency analysis tool. Instead of evaluating the DFT of a full-length sequence, the DFTs of several of its subsequences, ie. the STFT was computed for better time domain resolution by sliding the window by one entry in the sequence. It is a known fact that using the STFT increases resolution in time domain. For the first two methods, most of the literature asserts 351 to be the window size, especially for $\mathrm{C}$ elegans. But the authors have found that the window size varies with the method adopted and the DNA sequence analyzed. With the DFT used for frequency analysis, the window found to yield better result was 240 . The better result obtained with the single peaking IIR filter over the one described in [5] can be attributed to the higher attenuation seen in the stop band of the filter. The use of such a filter has given lesser noise without using the subsequent filter bank mentioned in [5]. DWT is a far more popular and potential signal processing tool today. However it has been used only for noise suppression here. Review of literature did not reveal a formal, randomized comparison of each of engineering methods mentioned here with other nonengineering approaches, hence such a comparison is not presented.

Table I: Tabulation of results. Exon locations of [GenBank:AF099922] as given in the NCBI database, and those obtained using the various DSP methods discussed here

\begin{tabular}{|c|c|c|c|c|c|c|}
\hline \multicolumn{7}{|c|}{ Exon locations obtained for $\mathrm{C}$ elegans } \\
\hline Binary method & EIIP method & Filter I & Filter 2 & $\begin{array}{l}\text { Reduced } \\
\text { computation } \\
\text { with Filter I }\end{array}$ & $\begin{array}{c}\text { Reduced } \\
\text { computation } \\
\text { with Filter } 2\end{array}$ & NCBI ranges \\
\hline $7921-8021(100)$ & $782 \mid-802 I(200)$ & $7921-8021(100)$ & $782 I-802 I(200)$ & $782 I-802 I(200)$ & $784|-802|(\mid 80)$ & $7947-8059(|1| 2)$ \\
\hline $9521-9821(300)$ & $9521-982 I(300)$ & $9521-9821(300)$ & $952 I-985 I(330)$ & $9521-987 \mid(350)$ & $9521-985 I(330)$ & $9548-9879(331)$ \\
\hline|| $02|-||22|(200)$ & || $02|-||22|(200)$ & || $02|-||22|(200)$ & $1092|-||22|(300)$ & || $02|-||27|(250)$ & $1092|-| 122 \mid(300)$ & III34-II397(263) \\
\hline$|232|-|252|(200)$ & $|242|-|262|(200)$ & $|242|-|262|(200)$ & $|232|-|254|(220)$ & $|232|-|252|(200)$ & $|232|-|254|(220)$ & |2485-I 2664(I79) \\
\hline$|428|-|462|(340)$ & $|422|-|462|(400)$ & $|422|-|462|(400)$ & $|422|-|462|(400)$ & $|422|-|462|(400)$ & $|422|-|462|(400)$ & $|4275-| 4625(350)$ \\
\hline
\end{tabular}




\section{Conclusion}

In this paper the authors have shown that appropriate alterations to the classical methods of exon prediction yields better results. For AF099922 C elegans, the window size for the binary and EIIP methods has been found to be 240, whereas for the digital filter method it is 450 , as against 351 mentioned in most of the literature. The window size thus should be selected depending on the method of analysis and also on the sequence analyzed. The filter1 as it is called in this paper is the common filter found in literature $[3,5]$; filter 2 has been designed by the authors. It's clear that this design is much better performance-wise as evident from the results. We have proposed the DWT to de-noise exon prediction, and it has been proved that it is the right tool for de-noising to be used with exon prediction algorithms.

\section{Competing interests}

The authors declare that they have no competing interests.

\section{Authors' contributions}

TPG carried out the work and drafted the manuscript under the close guidance of TT who conceived of the study, and participated in its design and coordination. Both authors have read and approved the final manuscript.

\section{Acknowledgements}

I would like to thank the authorities of the Department of Electronics, Cochin University of Science and Technology, Kerala, India, for permitting me to carry out this work under the guidance of Dr. Tessamma Thomas, the second author.

This article has been published as part of BMC Bioinformatics Volume II Supplement I, 20I0: Selected articles from the Eighth Asia-Pacific Bioinformatics Conference (APBC 2010). The full contents of the supplement are available online at http://www.biomedcentral.com//47|$2105 / I 1$ ? issue=SI.

\section{References}

I. Vaidyanathan PP: Genomics and Proteomics: A signal processor's tour. IEEE Circuits ad Systems Magazine, Fourth quarter 2004, 6-29.

2. Tiwari S, Ramachandran S, Bhattacharya A, Bhattacharya $S$ and Ramaswamy R: Prediction of probable genes by Fourier analysis of genomic sequences. CABIOS 1997, 13(3):263-270.

3. Trifonov EN and Sussman JL: The pitch of chromatin DNA is reflected in its nucleotide sequence. Proceedings of the Nat Acad Sci, USA 1980, 77:3816-3820.

4. Anastassiou D: Frequency-domain analysis of bio-molecular sequences. Bioinformatics 2000, 16(12): |073-108|.

5. Vaidyanathan PP and Yoon BJ: Gene and exon prediction using all-pass based filters. ieeexplore.org.

6. Fox TW and Carreira A: A Digital Signal Processing Method for Gene Prediction with Improved Noise Suppression. EURASIP Journal on Applied Signal Processing 2004, I: I08-I I 4.

7. George TP and Thomas T: Improvements in Gene Splicing and Gene Comparison for Anomaly Detection. Report of The M Tech Semester IV Project work 2009 April, done at Department of ElectronicsCochin University of Science And Technology, Kerala, India;

8. George TP and Thomas T: Exon Prediction Methods in Eukaryotes. Report of The M Tech Semester III Project work 2008
December, done at Department of ElectronicsCochin University of Science And Technology, Kerala, India;

9. Nair AS and Sreenadhan S: A coding measure scheme employing electron-ion interaction pseudopotential (EIIP). Bioinformation, Open access hypothesis 2006, 197-202.

10. Kakumani R, Devabhaktuni $V$ and Ahmad MO: Prediction of protein coding regions in DNA using a model based approach. IEEE Signal Processing Magazine 2009.

II. Proakis JG and Manolakis D: Digital Signal Processing.Prentice Hall of India, Pvt. Ltd; Fourth2007, 454-46I.

12. Soman KP and Ramachandran KI: Insights into Wavelets - From Theory to Practice.Prentice - Hall of India, Pvt. Ltd; Second2004.

\section{Publish with BioMed Central and every} scientist can read your work free of charge

"BioMed Central will be the most significant development for disseminating the results of biomedical research in our lifetime. "

Sir Paul Nurse, Cancer Research UK

Your research papers will be:

- available free of charge to the entire biomedical community

- peer reviewed and published immediately upon acceptance

- cited in PubMed and archived on PubMed Central

- yours - you keep the copyright
BioMedcentral 